Trauma Berufskrankh 2004 - 6 [Suppl 3] : S329-S333 DOI 10.1007/s10039-003-0740-1

Online publiziert: 28. Mai 2003

c) Springer-Verlag 2003

H.-U.Langendorff

Klinik für Unfall-, Hand- und Wiederherstellungschirurgie, Unfallklinik Dortmund

\title{
AC-Verletzungen: konservativ-funktionelle Therapie und Ergebnisse
}

Belastungsaufnahmen dienen dem Nachweis vertikaler Instabilitäten (- Abb. 2).Zum Nachweis einer horizontalen Instabilität, bei welcher sowohl eine Zerreißung des AC-Band-Apparats als auch der Trapezius- und Deltoideusmuskulatur vorliegt, eignet sich die Aufnahmetechnik nach Alexander [1]. Dabei werden die Schultern so weit als möglich nach vorne zusammengeführt, um so eine Überlagerung von Klavikula und Akromion zu provozieren (• Abb. 3). Diese Aufnahme ist insbesondere hilfreich, um zwischen Tossy-II- und Rockwood-IV-Verletzun- gen zu unterscheiden, da Letztere zwar definitionsgemäß, aber nicht zwangsläufig mit einer Ruptur des korakoklavikularen Bandapparats einhergehen und der klinische Befund sich ähnelt.

Die exakte Klassifikation bildet die Grundlage für die Wahl des weiteren Vorgehens. Dieses wird seit mehr als 100 Jahren heftig und $\mathrm{z}$. T.kontrovers diskutiert. Dies gilt insbesondere für die Tossy-III-Verletzungen, bei denen operative und konservative Verfahren konkurrieren und sich zyklischer Popularität erfreuen. akromioklavikularen Bandverbindungen oder knöchernem Abriss des Akromions führt (• Abb. 1), die korakoklavikularen Bandverbindungen bleiben dagegen intakt.

Das Verletzungsmuster reicht von der einfachen Kapsel-Band-Dehnung bis zur Zerreißung aller Bandstrukturen, Abriss der Muskulatur und Dislokation der Klavikula.

\section{Diagnostik}

Neben Anamnese und klinischem Befund kommt v. a.der radiologischen Diagnostik Bedeutung zu, anhand derer das Ausmaß der Verletzung abgeschätzt werden kann. Zugleich bildet sie auch die Basis für die Klassifikation der Verletzung.

Röntgenaufnahmen im a.-p.-Strahlengang mit um $30^{\circ}$ geneigter Röhre geben Aufschluss über evtl. knöcherne Begleitverletzungen oder arthrotische Veränderungen.

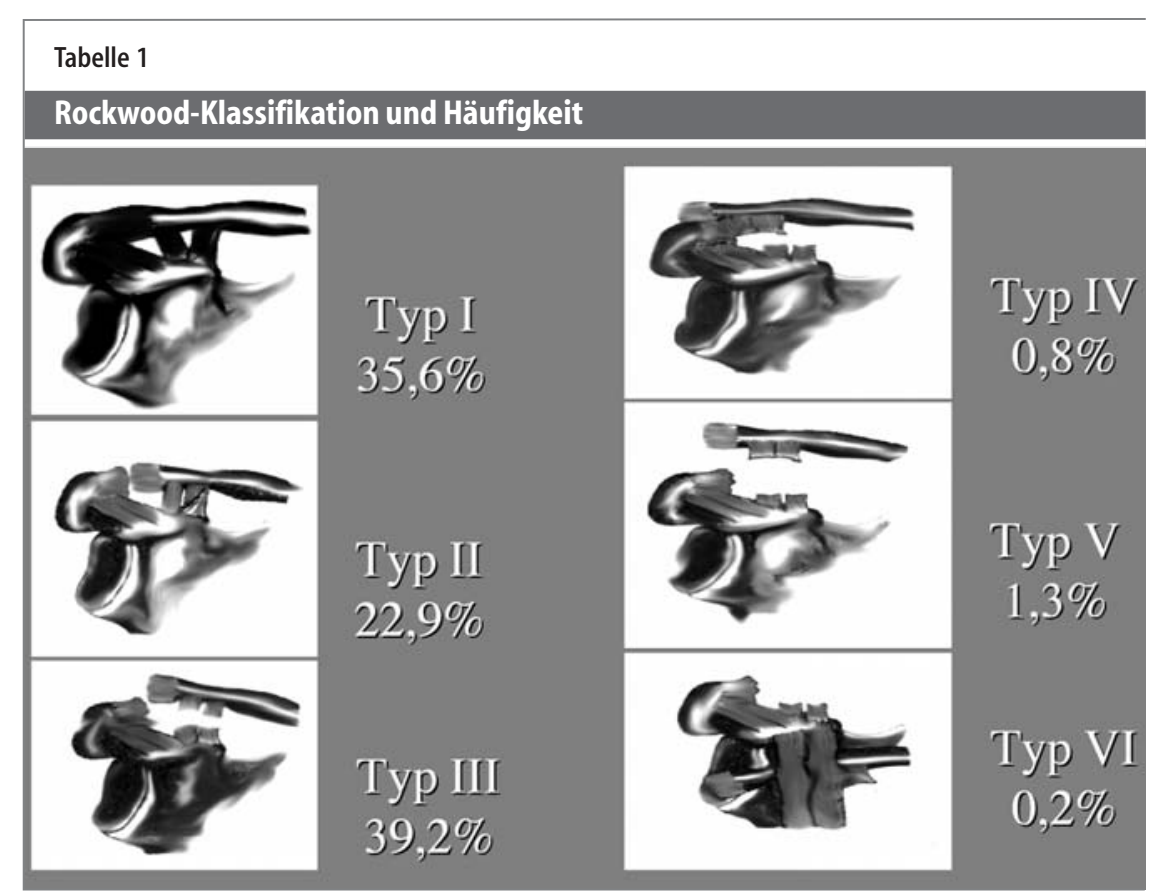



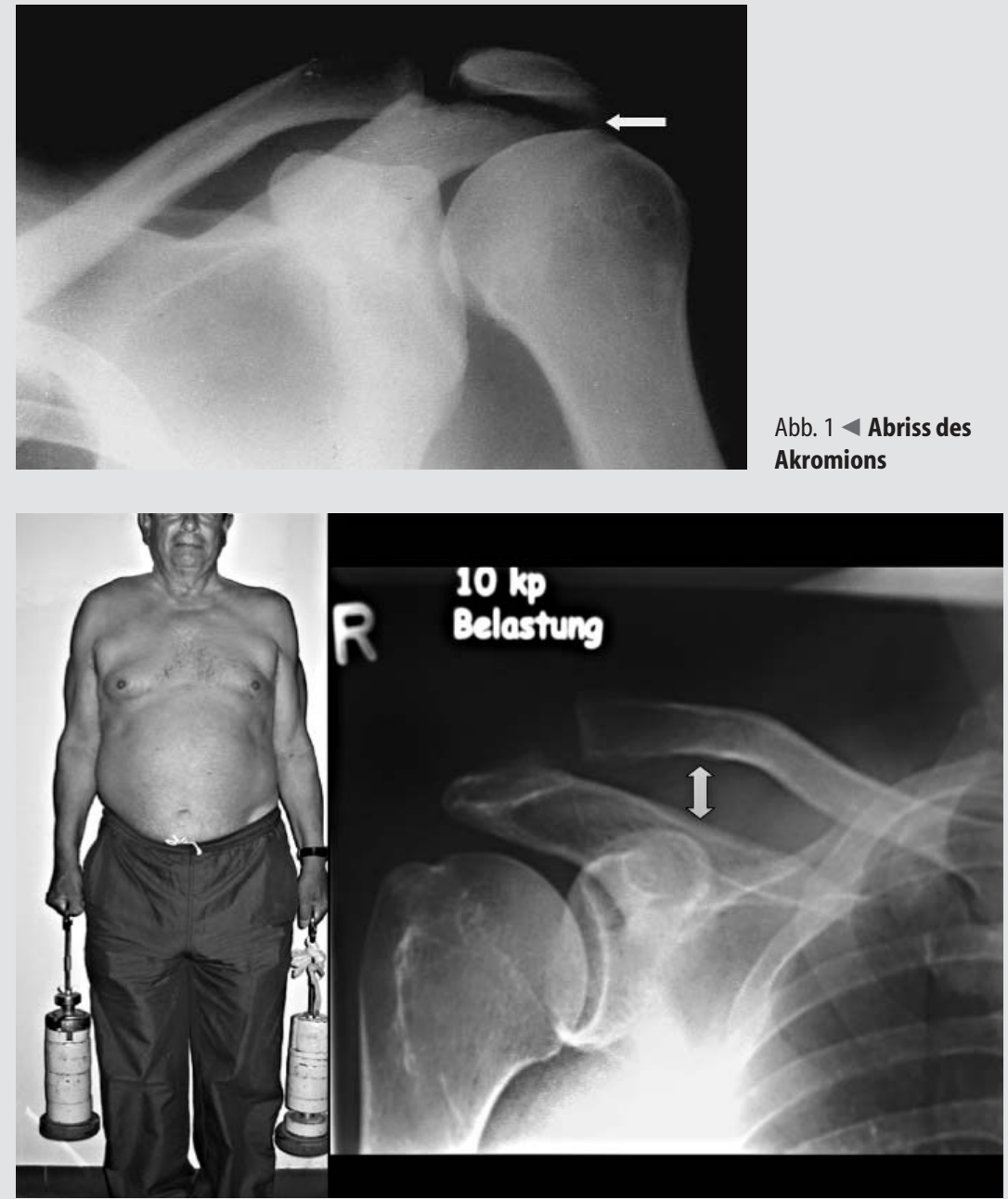

Abb. $2 \Delta$ Belastungsaufnahme

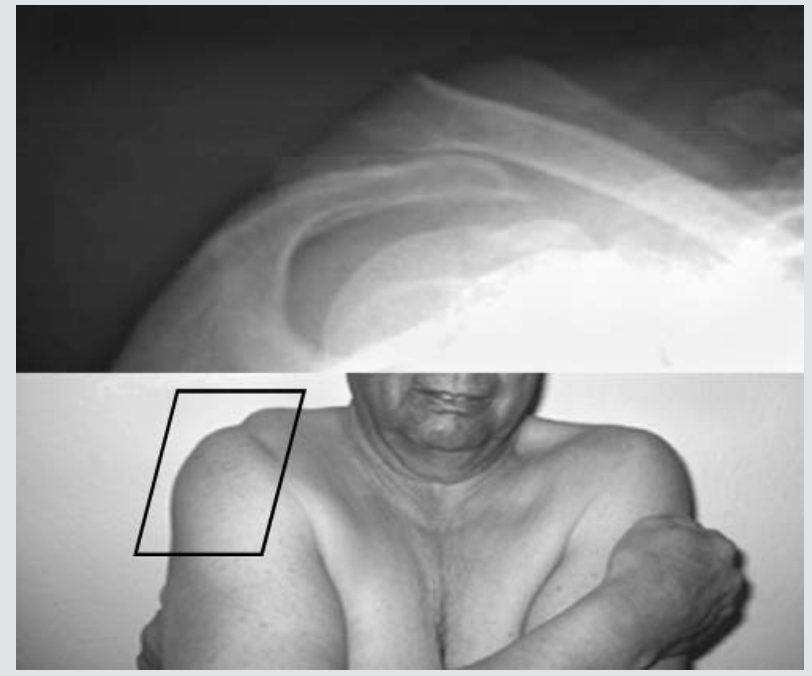

Abb. $1<$ Abriss des

Akromions

\section{Therapie}

\section{Konservative Therapie}

Konservative Verfahren reichen von monströsen Gipsverbänden bis zur „skillfull neglection“:

- Tapeverbände

- Schlingenverbände

- Schienen

- Gurte

- Abduktionsschienen

- Gipse

- Gilchrist-Verband

- „skillfull neglect“

\section{Operative Therapie}

Bislang wurden mehr als 50 verschiedene operative Verfahren zur Stabilisierung des AC-Gelenks angegeben, seitdem Cooper 1861 erstmals eine AC-Gelenk-Sprengung durch einen Silberdraht versorgte. Einige davon sind:

1. Arthrodese des AC-Gelenks

- Versorgung mit Schrauben

- Versorgung mit Platten

- Versorgung mit Drähten

- Versorgung mit Nähten jeweils mit/ohne Bandrekonstruktion

2. Fixation des cc-Bandes - Versorgung mit Schrauben

- Versorgung mit Draht

- Versorgung mit Faszie

- Versorgung mit Sehnen

- Versorgung mit Nähten jeweils mit/ohne ac-Band-Rekonstruktion

3. distale Klavikularesektion mit/ohne cc-Band-Rekonstruktion durch

- Versorgung mit Faszie

- Versorgung mit Naht

- Versorgung mit ac-Band-Transfer

Abb. 34 Aufnahmetechnik nach Alexander [1] 
werden in $84 \%$ aller Kliniken Tossy-IIIVerletzungen überwiegend operativ versorgt, obgleich $37 \%$ der Kliniken davon überzeugt waren, dass eine konservative Therapie mindestens gleichwertig oder sogar überlegen sei.

Weitgehend Einigkeit besteht dahingehend, Tossy-I- und -II-Verletzungen konservativ funktionell und alle höhergradigen Verletzungen vom Typ Rockwood IV-VI operativ zu versorgen.

Tossy-I-Verletzungen, bei denen ja nur eine Kapsel-Band-Dehnung vorliegt, bedürfen nur einer kurzfristigen Ruhigstellung des Schultergelenks für wenige Tage bis zum Abklingen des akuten Schmerzes. Lokale Eisanwendungen und ggf. milde Analgetika erhöhen den Patientenkomfort.

Gleiches gilt im Prinzip auch für die Tossy-II-Verletzungen, trotz vollständiger Zerreißung des ac-Bandes und meist auch der Gelenkkapsel mit Subluxation der Klavikula. Meist ist jedoch der Zeitraum der Ruhigstellung bis zu vollkommenen Schmerzfreiheit länger. Sportliche Aktivitäten sollten für 4-6 Wochen eingeschränkt werden, um nicht bei einem erneuten Sturz eine Zerreißung der cc-Bänder zu riskieren, wodurch dann die TossyII-Verletzung zwangsläufig in eine TossyIII-Verletzung übergehen würde.

Reichkendler et al. [13], die operativ und konservativ behandelte Tossy-II-Verletzungen miteinander verglichen, konnten bezüglich des Constant-Murley-Scores, der Schmerzhaftigkeit und der Sportfähigkeit keine Überlegenheit eines operativen Verfahrens feststellen.

Umstritten ist die Therapie bei TossyIII-Verletzungen. Bereits Hippokrates, auf den die erste Beschreibung einer vollständigen AC-Gelenk-Sprengung zurückgeht, empfahl zügelnde Verbände. Gleichwohl erkannte er bereits damals, dass eine Retention hierdurch nicht dauerhaft gesichert werden konnte und dennoch das Ergebnis meist gut war. Galen, der sich selbst beim Ringen eine derartige Verletzung zugezogen hatte, berichtete dass er einen derartigen Zügelungsverband als so lästig und unbequem empfand, dass er ihn nach wenigen Tagen entfernte und weiter seinem Kampfsport frönte. Trotz vielfältiger Modifikationen derartiger Verbände in der Folgezeit - und es existieren

Trauma Berufskrankh 2004 · 6 [Suppl 3] : S329-S333

DOI 10.1007/s10039-003-0740-1

(c) Springer-Verlag 2003

H.-U.Langendorff

\section{AC-Verletzungen: konservativ-funktionelle Therapie und Ergebnisse}

\section{Zusammenfassung}

Schultereckgelenkverletzungen gehören mit über 10\% zu den zweithäufigsten Verletzungen des Schultergürtels. Während AC-Gelenk-Verletzungen vom Typ Tossy I und II generell konservativ-funktionell und Verletzungen vom Typ Rockwood IV-VI operativ versorgt werden, ist die Therapie der Tossy-III-Verletzungen umstritten. Hier konkurrieren mehr als 35 verschiedene konservative Methoden mit über 50 operativen Verfahren. Unabhängig von der Verfahrenswahl zeigen diese vergleichbar gute funktionelle Ergebnisse. Abgesehen von einem verbleibenden Klavikulahochstand, der auch durch redressierende Verbände meist nicht vermieden werden kann, konnte die postulierte Verminderung der Schulterkraft nach konservativer Behandlung nicht

\section{Acromioclavicular injuries: conservative-functional therapy and results}

\section{Abstract}

Injuries involving the acromoclavicular joint account for more than $10 \%$ of all injuries to the shoulder girdle, making them the second most frequent type of shoulder injury. When categorized as Tossy I or II such injuries are generally treated conservatively, whereas Rockwood IV-VI injuries are treated operatively. There is still no agreement on the best way of treating Tossy class III injuries. More than 35 conservative treatments have been proposed, and over 50 operative methods. Regardless of the method of treatment applied, relatively good functional results are obtained. Persisting prominence of the clavicle cannot reliably be reduced by strapping, but the reduced shoulder strength that has been claimed to follow conservative treatment has durch Kraftmessungen verifiziert werden. Darüber hinaus sind nach operativer Behandlung die Rehabilitation sowie die Sport- und Arbeitsunfähigkeit länger, die Rate an Komplikationen ist deutlich höher und mit erhöhten Kosten verbunden..,skillfull neglection" der Tossy-III-Verletzungen mit kurzfristiger Ruhigstellung des Schultergelenks, Kryotherapie und funktioneller Behandlung scheint auch bei körperlich schwer arbeitenden oder sportlich aktiven Patienten die Therapie der Wahl zu sein.

\section{Schlüsselwörter}

Akromioklavikulargelenkverletzung · Operative Therapie · Konservative Therapie · Ergebnisse

not been verified by strength testing. Furthermore, after surgery rehabilitation takes longer, the period of inability to work and to engage in sporting activities is extended, and the complication rate is higher, in addition to which the costs of surgical treatment are higher. "Skillful neglect" of Tossy I-III injuries combined with brief immobilization of the shoulder joint, cryotherapy and functional treatment seems to be the method of choice even in patients whose work involves hard physical labour and in active athletes.

\section{Keywords}

Injuries to the acromioclavicular joint · Operative and conservative therapy · Results 
Tabelle 2

\section{Behandlungsergebnis nach Tossy-III-Verletzungen, aus Phillips et al. [10]}

\begin{tabular}{|llll|}
\hline Ergebnis & Studien & $\begin{array}{l}\text { Operative } \\
\text { Behandlung }\end{array}$ & $\begin{array}{l}\text { Konservative } \\
\text { Behandlung }\end{array}$ \\
\hline Schmerz: keiner/gering & 7 & $93 \%$ & $96 \%$ \\
Bewegungsausmaß: normal & 6 & $86 \%$ & $95 \%$ \\
Kraft: normal & 7 & $87 \%$ & $92 \%$ \\
\hline
\end{tabular}

Tabelle 3

Behandlungsdauer nach Tossy-III-Verletzungen, nach Press et al. [11]

\section{Dauer}

Immobilisation [Wochen]

Arbeitsunfähigkeit [Monate]

Sportunfähigkeit [Monate]

Schmerzfreiheit [Monate]
Operative Behandlung

6,2

2,6

6,4

5,5
Konservative Behandlung

2,7

0,8

3,5

6,5 mindestens 35 Varianten - waren sie allesamt meist wenig erfolgreich.

Die Gründe hierfür waren unterschiedlich. Mangelnde Compliance, Druckulzera und Unbequemlichkeit spielten eine Rolle. Darüber hinaus ist eine Ruhigstellung eines Schultergelenks für 6 Wochen mit der zu erwartenden Einsteifung nicht zu vertreten.

So ist es nicht verwunderlich, dass operative Verfahren von einer Vielzahl von Autoren bevorzugt werden. Von ihnen werden, außer den genannten Gründen, kosmetische und v. a. auch ein drohender Kraftverlust argumentativ ins Feld geführt. Man mag geteilter Meinung sein, ob eine hochstehende Klavikula schöner ist als eine evtl. hypertrophe Narbe. Schwerwiegender ist das Argument des Kraftverlusts, insbesondere bei körperlich schwer arbeitenden oder sportlich aktiven Patienten.

Fremerey et al. [7] gingen dieser Frage anhand objektiver Kraftmessungen in verschiedenen Gelenkpositionen nach. Sie konnten zeigen, dass ein verbleibender Klavikulahochstand nicht zu einer Kraftminderung führte und sich auch im Gesamtergebnis keine Überlegenheit operativ gegenüber konservativ behandelter Tossy-III-Verletzungen ergab.

$\mathrm{Zu}$ einem ähnlichen Ergebnis kamen Press et al. [11] bei isokinetischen Kraftmessungen operativ und konservativ behandelter Tossy-III-Verletzungen. Auch Tibone et al. [16], die umfangreiche Kraftmessungen am Cybex-Dynamometer durchführten, widerlegten die postulierte Kraftminderung bei Wurfbewegungen. Schlegel et al. [15] sahen in einer prospektiven Untersuchung über $1 \mathrm{Jahr}$ nur einen kurzfristigen Kraftverlust gegenüber der unverletzten Seite von $17 \%$. Zusammenfassend muss man also davon ausgehen, dass das Argument des Kraftverlusts nicht haltbar ist.

Auch auf die Entstehung einer posttraumatisch-degenerativen Veränderung wie Arthrose, Verkalkungen oder Lyse der distalen Klavikula hat die Wahl des Therapieverfahrens keinen Einfluss. Diese Veränderungen treten mit einer Häufigkeit von $14-92 \%$ nach operativer und $41-58 \%$ nach konservativer Therapie auf, ohne dass sie nachweislich Auswirkungen auf das subjektive oder funktionelle Ergebnis hatten. Tendenziell besteht vielmehr der Eindruck, dass eine verbleibende Subluxation eher arthrosefördernd, eine vollständige Luxation hingegen eher arthroseprotektiv ist, da keine direkte Artikulation der Gelenke mehr besteht. Sollte sich dennoch eine schmerzhafte, funktionsbehindernde Arthrose einstellen, kann dann immer noch eine laterale Klavikularesektion in der Regel mit gutem Ergebnis durchgeführt werden.

In einer Metaanalyse von über 600 Publikationen konnten Phillips et al. [10] zeigen, dass, bezogen auf die verwertbaren Studien, keine Unterschiede im Behandlungsergebnis zwischen konservativ und operativ behandelten Patienten bestanden
(- Tabelle 2). Auch Bäthis et al. [2] kamen bei einer Auswertung von 370 Publikationen nach den Kriterien der, „evidence based medicine“ zu einem ähnlichen Ergebnis. Insgesamt kann in nahezu $80 \%$ der TossyIII-Verletzungen unabhängig von der Verfahrenswahl mit einem guten Ergebnis gerechnet werden.

Aus den dargestellten Gründen vertreten auch wir die „skillfull neglection“ der Tossy-III-Verletzung. Ähnlich wie bei geringergradigen Verletzungen erfolgt nur eine kurzfristige Ruhigstellung der Schulter im Gilchrist-Verband, ergänzt durch Kryotherapie und Analgetika. In aller Regel hat der Patient nach 2-3 Wochen seinen vollen Bewegungsumfang wieder erreicht. Physiotherapeutische Maßnahmen sind in aller Regel vollkommen entbehrlich.

Wesentlich ist dabei jedoch, den Patienten detailliert über seine Verletzung und das zu erwartende kosmetische Erscheinungsbild aufzuklären und gemeinsam mit ihm auch die Vor- und Nachteile eines operativen oder konservativen Vorgehens zu erörtern.

In Zeiten hohen wirtschaftlichen Drucks erlangen neben der Qualität der Versorgung auch Therapiekosten einen immer höheren Stellenwert. Eine konservative Therapie kann stets ambulant erfolgen und verursacht damit weit geringere Kosten als eine Operation mit stationärem Aufenthalt, Zweiteingriff und der möglichen Komplikationen. Auch ist die Dauer der Arbeitsunfähigkeit nach konservativem Vorgehen sehr viel kürzer (- Tabelle 3).

\section{Fazit für die Praxis}

Zusammenfassend muss man daher zu dem Schluss kommen, dass die konservative Therapie bei Tossy-Verletzungen Grad I-III das Verfahren der Wahl und der operativen Therapie mindestens gleichwertig und allemal ökonomischer ist. Schwere körperliche Arbeit und sportliche Aktivitäten stellen keinen Grund für eine Operationsindikation dar. Unabhängig davon bleibt ist die Operation bei Verletzungen vom Typ Rockwood IV-VI indiziert. Die Behandlungsstrategie und deren Auswirkungen sollten jedoch stets mit dem Patienten besprochen werden, um hernach die Therapieentscheidung fällen zu können. 


\section{Korrespondierender Autor}

Prof.Dr.H.-U.Langendorff

Klinik für Unfall-, Hand-

und Wiederherstellungschirurgie,

Unfallklinik Dortmund,

Münsterstraße 240,44145 Dortmund,

E-Mail:UnfallklinikDo@aol.com

\section{Literatur}

1. Alexander OM (1954) Radiography of the acromiclavicular articulation. Med Radiogr Photogr 30: 34-39

2. Bäthis H, Tingart M, Bouillon B, Tiling T (2000) Konservative oder operative Therapie der Schultereckgelenksverletzung - was ist gesichert? Eine systematische Literaturanalyse nach Kriterien der ${ }_{\text {, }}$ evidence based medicine". Chirurg 71: 1082-1089

3. Bäthis H, Tingart M, Bouillon B, Tiling T (2001) Stand der Therapie der Schultereckgelenkverletzung - Ergebnisse einer Umfrage an Unfallchirurgischen Kliniken in Deutschland. Unfallchirurg 104: 955-960

4. Bosch U, Fremerey RW (2002) AC-Gelenkverletzungen - Konservative oder operative Behandlung der AC-Gelenkverletzungen.Zentralbl Chir 127: 203-206

5. Clarke HD, McCann P (2000) Acromoiclavicular joint injuries. Orthop Clin North Am 31: 177-187

6. Edelson JG (1996) Patterns of degenerative change in the acromioclavicular joint. J Bone Joint Surg $\mathrm{Br} 78$ 242-243

7. Fremerey RW, Lobenhoffer P, Ramacker K, Gerich T, Skutek M, Bosch U (2001) Akute AC-Gelenksprengung operative oder konservative Therapie? Unfallchirurg 104: 294-299

8. Larsen E, Bjerg-Nielsen A, Christensen P (1986) Conservative or surgical treatment of acromioclavicular dislocation.J Bone Joint Surg Am 68: 552-555

9. McFarland EG, Blivin SJ, Doehring CB et al. (1997) Treatment of grade III acromioclavicular separations in professional throwing athletes: results of a survey. Am J Orthop 26: 771-774

10. Phillips AM, Smart C, Groom AFC (1998) Acromioclavicular dislocation. Conservative or surgical therapy. Clin Orthop 353: 10-17

11. Press J, Zuckerman JD, Gallagher M, Cuomo F (1997) Treatment of grade III acromoclavicular separations. Operative versus nonoperative management. Bull Hosp Joint Dis 56: 77-83

12. Rawes ML, Dias JJ (1996) Long term results of conservative treatment for acromioclavicular dislocation.J Bone Joint Surg Br 78: 410-412

13. Reichkendler M, Rangger C, Dessl A, Ulmer H (1996) Vergleich und Ergebnis von Schultereckgelenkverletzungen II. und III. Grades. Unfallchirurg 99: 778-783

14. Riand N, Sadowski C, Hoffmeyer P (1999) Disjunctions acromio-claviculaires récentes. Acta Orthop Belg 65: 393-403

15. Schlegel TF, Burks RT, Marcus RL, Dunn HK (2001) A prospective evaluation of untreated acute grade III acromiclavicular separations. Am J Sports Med 29: 699-703

16. Tibone J, Sellers R, Tonino P (1992) Strength testing after third-degree acromoclavicular dislocations. Am J Sports Med 20: 328-331

17. Wojtys EM, Nelson G (1991) Conservative treatment of grade III acromioclavicular dislocations. Clin Orthop 268: $112-119$
Die Zeitschrift Trauma und Berufskrankheit steht für eine ganzheitliche Information zu Fragen aus dem Bereich der Unfall- und Wiederherstellungschirurgie, der Berufserkrankungen sowie für die Bearbeitung aller Aspekte der gesetzlichen Unfallversicherung.

In diesem Rahmen werden Themen der Unfallverhütung, der Diagnostik, der operativen und konservativen Therapie, der Folgeschäden und Folgeeingriffe, der Rehabilitation sowie gesetzliche Grundlagen, Kriterien der Entschädigung und Aspekte der Umschulung u. a. behandelt.

Um diese Themenvielfalt aktuell und praxisnah in der Zeitschrift abzubilden, möchten wir Sie ermuntern, interessante Arbeiten bei uns einzureichen.

Im Vordergrund stehen dabei Beiträge, die sich mit folgenden Themen befassen:

- Berufskrankheiten

- BG-Heilverfahren

- Arbeitsmedizin

- Begutachtung

- Rehabilitation

- Qualitätssicherung

- Der besondere Fall

- Forschung

- Spezielle Techniken, Innovationen

- Arzneimitteltherapie

- Tipps \& Tricks

Bitte schicken Sie Ihre Manuskripte in 2-facher Ausfertigung inkl. Diskette (bzw. Datei per E-Mail) an Herrn Prof.Dr. D. Wolter (koordinierender Schriftleiter) oder

Herrn Priv.-Doz.Dr.C.Jürgens,

Ärztlicher Direktor des Berufsgenossenschaftlichen Unfallkrankenhauses,

Bergedorfer Straße 10,21033 Hamburg,

Tel.: 040/7306-2701, Fax: 040/7306-2703,

E-Mail:ch.juergens.bukh@t-online.de

Ihre Arbeit wird anschließend von wenigstens zwei Gutachtern geprüft.

Die Schriftleitung behält sich das Recht vor, Manuskripte abzulehnen.

Unsere Hinweise für Autoren finden Sie im Internet unter

TraumaundBerufskrankheit.springer.de 